\title{
PROBABILIDADES DE OCURRENCIA DE DESCARGAS ELÉCTRICAS ATMOSFÉRICAS PARA LA CUENCA DEL RÍO MANTARO - PERÚ, UTILIZANDO BANDAS DE VAPOR DE AGUA E INFRARROJA DEL SATÉLITE GOES
}

\section{PROBABILITIES OF OCCURRENCE OF ATMOSPHERIC ELECTRIC DISCHARGES FOR THE MANTARO RIVER BASIN - PERU, USING GOES SATELLITE INFRARED AND WATER VAPOR BANDS}

\author{
Weidi Flores ${ }^{1,2}$, Delia Acuña ${ }^{3}$, Victoria Calle $e^{1,4}$ y Jerónimo García ${ }^{1,5}$
}

\begin{abstract}
Resumen
Se encontraron relaciones estadísticas para el cálculo de probabilidades de ocurrencia de rayos, tomando como base la diferencia de temperatura de brillo de la banda de vapor de agua e infrarrojo del satélite GOES y datos de ubicación de rayos del STARNET para las comunidades campesinas de Marcapomacocha (4 $479 \mathrm{msnm}$ ) y Huayao (3 $350 \mathrm{msnm}$ ) ubicadas en la cuenca del río Mantaro, en la sierra central de Perú. La aplicación de las relaciones en los algoritmos de probabilidades permitió hacer seguimiento a los sistemas de nubes que presentaron características definidas para la ocurrencia de descargas eléctricas atmosféricas. Los resultados de esta novedosa técnica demostraron que, realizando los adecuados ajustes en la precisión de detección de rayos, se pueden llegar a obtener óptimos resultados utilizando mayor cantidad de datos de ambos sistemas de detección e implementar un sistema de seguimiento de ocurrencia de descargas eléctricas atmosféricas, aportando información relevante ante estos eventos meteorológicos extremos, siendo muy útil para las diversas actividades económicas en el país.

Palabras clave: descargas eléctricas de origen atmosférico, temperatura de brillo, algoritmo, cálculo de probabilidades, satélite meteorológico, pronóstico de corto plazo.
\end{abstract}

\section{Abstract}

Statistical summaries were found in order to calculate likelihood of lighting occurrence. They were based on the difference of bright temperature from the water vapor band and satellite's infrared GOES, and location lightning data from STARNET for countrymen communications of Marcapomacocha (4 479 meters above sea level) and Huayao (3 350 meters above sea level) located in the basin of Mantaro, from the highlands of central Peru. The usage of statistical summaries in algorithms let monitor the cloud systems that offer defined characteristics to electrical atmospheric discharge occurrence. The results of this innovative technique proved that doing the correct setting adjustments on the precision of lightning detection, optimal results can be reached using more data from both detection systems. Besides, adding a monitor system of electrical atmospheric discharge occurrence could contribute with relevant information to respond to these extreme meteorological events being useful for the economic activities in the country.

Key words: electrical discharge of atmospheric origin, brightness temperature, algoritm, probability calculation, meteorogical satellite, short term forecast.

\section{Introducción}

Una tormenta se define como una o más descargas repentinas de electricidad que se manifiestan con un destello de luz y con ruido o un estruendo sordo; las tormentas se asocian a nubes Cumulonimbus y suelen ir acompañadas de precipitación que, cuando alcanza el suelo, lo hace en forma de chubasco de lluvia, nieve, nieve granulada, cellisca o granizo (WMO, 2017).

Según el comité de expertos en relámpagos y clima de la OMM (Organización Meteorológica Mundial, WMO en inglés), definen a la descarga eléctrica como una serie de procesos que se producen de forma continuada, cuyo relámpago puede recorrer largas distancias respecto de la tormenta que las genera (WMO, 2020). La descarga preliminar puede desarrollarse en cualquier región de la nube donde el campo eléctrico desarrollado venza el dieléctrico del aire.

Para el caso del parámetro dinámico de las nubes, Kumjian et al. (2014) indican que el flujo ascendente de aire es el responsable de la elevación de las gotas de agua líquida superenfriadas hasta los niveles superiores de la nube, lugar donde estos hidrometeoros forman los cristales de hielo y granizo, evidenciando la importante relación del flujo ascendente en la electrificación de las nubes. Así también, algunos autores (Lang \& Rutledge, 2002; Tessendorf et al., 2005; Deierling \& Petersen, 2008) indican que la densidad de las descargas eléctricas está asociada con la intensidad del flujo ascendente indicando que un flujo ascendente más 
intenso favorece el incremento de las colisiones entre los hidrometeoros, el cual ocasiona un mayor intercambio de cargas y de esta forma una mayor electrificación de la nube. Wallace \& Hobbs (2006) mencionan que las tormentas ordinarias son originadas en nubes aisladas del tipo cumulonimbos producidas por convección en una masa de aire inestable al paso de un frente frio o líneas de inestabilidad. Falcón \& Quintero (2009) mencionan que los electrometeoros, como los rayos son manifestaciones de la ruptura dieléctrica del aire ante la gran diferencia de potencial entre dos puntos en la atmosfera. La microfísica asociada de los electrometeoros parece estar relacionada también a las propiedades físicas y químicas de la atmósfera local de ocurrencia. Keith \& Saunders (1990) y Baker et al. (1995), encontraron que la actividad eléctrica de las tormentas está relacionada con la concentración y el tamaño de los cristales de hielo, así como de la distribución de las gotas de agua líquida superenfriadas (Saunders et al., 1991). De la misma forma algunos autores (Herzegh \& Jameson, 1992; Homeyer \& Kumjian, 2015; Snyder et al., 2015) observaron, en sistemas convectivos, la presencia de gotas de agua superenfriadas por encima de la isoterma de $0 \quad{ }^{\circ}$ C. Mattos et al. (2017) observaron este comportamiento antes y durante el inicio de la actividad eléctrica de las tormentas eléctricas, sugiriendo que las gotas de agua líquida superenfriadas al congelarse forman cristales de hielo y granizo cónico, los cuales son necesarios para generar la electrificación de la nube.

Machado et al. (2009) relacionan las descargas eléctricas con las nubosidades del tipo convectivo que atraviesan el nivel de la tropopausa; para tal efecto, combinan las imágenes de los canales infrarrojo y las del vapor de agua del satélite GOES 12 y las relacionan con la ocurrencia de descargas eléctricas atmosférica de nube a tierra. Vila et al. (2008) desarrollaron y validaron un algoritmo para el seguimiento y pronóstico de radiación y características morfológicas de los sistemas convectivos de mesoescala (SCM), a través de sus ciclos de vida completos, utilizando información del canal térmico del satélite GOES; se aplicó la herramienta de pronóstico de corto plazo para evaluar el desplazamiento de los complejos convectivos de mesoescla (MCS) y la evolución del tamaño en la cuenca del Río de la Plata en Sudamérica con intervalos de tiempo de 30 hasta 120 minutos. Mecikalski \& Bedka (2006) y Mecikalski et al. (2008) mostraron que los atributos físicos asociados con el desarrollo de nubes cumulonimbos pueden ser monitoreados mediante diferencias de canales o mediante el empleo de bandas espectrales específicas. Arellano \& Escalante (2014) desarrollaron una metodología para la estimación del potencial de tormentas basada en el empleo de patrones conocidos de estas durante periodos anteriores, asociando datos medidos en superficie e información interpretada de temperatura de brillo procedente de imágenes de satélite correspondientes al espectro infrarrojo (IR) en la banda $10.7 \mu \mathrm{m}$ (canal 4), provenientes de los satélites GOES 11 y GOES 12. En general, el procesamiento de las imágenes IR consistió en la transformación de los valores de píxeles de las imágenes ( $\begin{array}{lll}0 & \text { a } 255) \text { a }\end{array}$ temperaturas de brillo en ${ }^{\circ} \mathrm{C}$.

\section{Materiales y métodos}

Las zonas de estudio para la realización de esta investigación corresponden a la cuenca del río Mantaro, habiéndose seleccionado por ser una zona de gran desarrollo de diversas actividades económicas como la agricultura, ganadería, comercio, etc., que conglomera alta densidad de habitantes; asimismo, por contener una red de estaciones meteorológicas en comparación con otras zonas del país, tomando como referencia a las estaciones meteorológicas del Servicio Nacional de Meteorología e Hidrología y como puntos focales a aquellas ubicadas en las comunidades campesinas de Marcapomacocha y Huayao, localizadas en las provincias de Yauli y Chupaca, respectivamente, en el Departamento de Junín. Estas localidades fueron seleccionadas con la intención de contrastar los diferentes aspectos de la atmósfera ante la ocurrencia de descargas eléctricas atmosféricas en sectores altos y medios respectivamente de la cuenca del rio Mantaro en los andes centrales de Perú. (Tabla 1; Figura 1).

Tabla 1. Localización de estaciones meteorológicas.

\begin{tabular}{llll}
\hline $\begin{array}{l}\text { Estación } \\
\text { Meteorológica }\end{array}$ & $\begin{array}{l}\text { Latitud } \\
\text { Sur }\left({ }^{\circ}\right)\end{array}$ & $\begin{array}{l}\text { Longitud } \\
\text { Oeste }\left({ }^{\circ}\right)\end{array}$ & $\begin{array}{l}\text { Altitud } \\
(\mathbf{m s n m})\end{array}$ \\
\hline Carhuamayo & -10.97 & -76.06 & 4130 \\
Huasahuasi & -11.26 & -75.62 & 2751 \\
Tarma & -11.39 & -75.69 & 3000 \\
Santa Ana & -12.01 & -75.22 & 3295 \\
Viques & -12.16 & -75.24 & 3186 \\
Jauja & -11.79 & -75.49 & 3186 \\
Huayao & -12.04 & -75.34 & 3350 \\
Yantac & -11.33 & -76.40 & 4684 \\
Marcapomacocha & -11.41 & -76.33 & 4479 \\
Yauli & -11.67 & -76.08 & 4100 \\
La Oroya & -11.52 & -75.90 & 4040 \\
Carhuacayan & -11.20 & -76.28 & 4150 \\
\hline
\end{tabular}

Se utilizó inicialmente información de temperatura de brillo de la banda de vapor de agua (banda 3 ) e infrarroja (banda 4) del satélite GOES 13 entre junio del 2015 a noviembre del 2017, para la elaboración de algoritmos de cálculos de probabilidades con el uso del software estadístico R-Studio; posteriormente, al entrar en operaciones el satélite GOES 16, se utilizó información de sus bandas equivalentes de vapor de agua (banda 9) e infrarrojo (banda 14) con las cuales se realizaron cálculos de las probabilidades de ocurrencia de descargas eléctricas atmosféricas utilizando para este efecto las localizaciones geográficas (latitud y longitud) de descargas eléctricas atmosféricas 
acontecidas en las inmediaciones de las estaciones meteorológicas seleccionadas para fechas específicas. Georreferenciación de puntos de descargas eléctricas atmosféricas

Se seleccionaron los eventos de descargas eléctricas atmosféricas acontecidos entre junio del año 2015 y noviembre del 2017 del portal web del Sferics Timing and Ranging Network (STARNET, 2017) accediéndose a la información de localización de descargas eléctricas atmosféricas (latitud y longitud) a nivel de la cuenca del río Mantaro, considerándose aquellos eventos acontecidos hasta los $10 \mathrm{~km}$ alrededor de las estaciones meteorológicas en las zonas de interés y aproximadamente al momento de emisión de las imágenes de satélite respectivas. Para este fin se utilizó la técnica de Diferencia de Tiempo de Llegada (ATD) (Lee, 1986) que conlleva a una localización del rayo sustancialmente mejorada; para este efecto se consideraron aquellos errores de ATD menores a $20 \mu \mathrm{s}$, que significan los menores errores de localización de rayos, siendo estos menores a $15 \mathrm{~km}$ dentro de la periferia de la red de detección del STARNET.

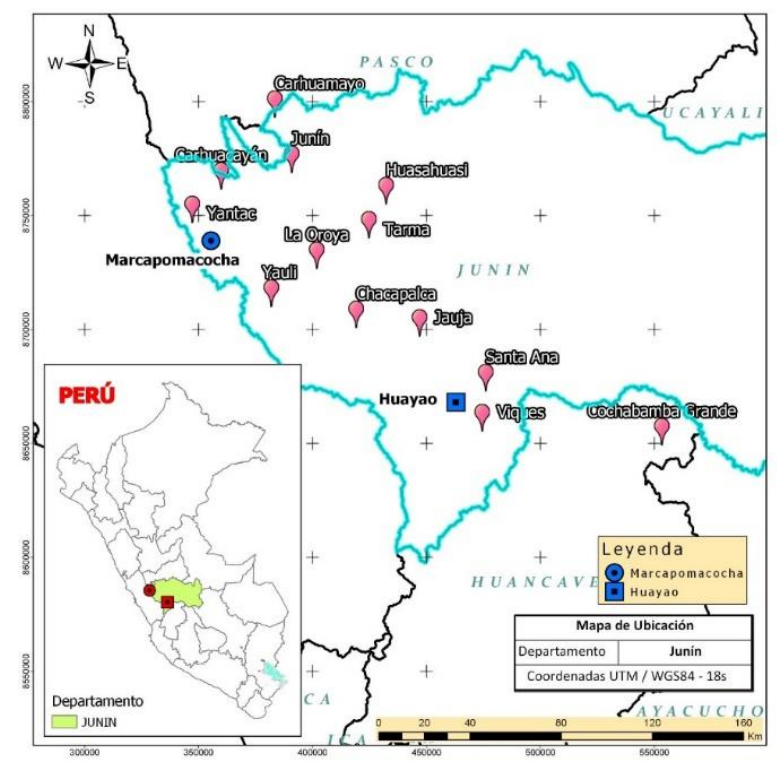

Figura 1. Localización de estaciones meteorológicas.

Diferencia de temperatura de brillo entre la banda de vapor de agua e Infrarroja (GOES 13 y GOES 16)

Inicialmente se utilizaron los archivos en formato NetCDF de las imágenes del satélite GOES 13 para la banda 3 (vapor de agua) y banda 4 (infrarroja); extrayendo los datos de temperatura de brillo de las áreas que coincidieron con la localización de descargas eléctricas atmosféricas (en el tiempo y el espacio) de los reportes del portal STARNET con el fin de asociar la ocurrencia y las localizaciones de las mismas con los sistemas nubosos que originaron las descargas eléctricas atmosféricas; los tiempos de ocurrencia de ambos sistemas fueron sincronizados tomando como referencia el momento de ocurrencia de los rayos con el momento de emisión de la imagen de satélite respectiva, con 20 minutos antes o después de la misma, tomando en consideración el Tiempo Universal Coordinado (UTC) en ambos sistemas de detección. Las diferencias positivas entre la temperatura de brillo del vapor de agua y la infrarroja son solo posibles cuando las nubes de convección profunda penetran la estratósfera. Mediante los estudios de observación se revela que las descargas eléctricas están asociadas con presencia de grandes partículas de hielo y fuertes corrientes ascendentes en la región de mezcla $\left(0{ }^{\circ} \mathrm{C}\right.$ y $20^{\circ} \mathrm{C}$ ) (Baker et al., 1995; Petersen \& Rutledge, 1998). Por lo tanto, las nubosidades de convección profunda y muy extendidas son responsables de las altas tasas de descargas eléctricas (Abdoulaev et al., 2001).

Se tomaron los valores extraídos tanto de la banda 3 y banda 4 del satélite GOES 13 y se obtuvieron las diferencias de temperatura de brillo (TB3 - TB4), las cuales se calcularon para los eventos donde hayan ocurrido descargas eléctricas atmosféricas entre el periodo establecido para cada evento en las diversas zonas de la cuenca en estudio. Para cada imagen pixelada de diferencias de temperatura de brillo del satélite, se encontraron las ocurrencias de descargas eléctricas atmosféricas de la base de datos del STARNET con 20 minutos antes o después del momento reportado de la imagen de satélite respectiva y en un radio máximo de $10 \mathrm{~km}$ alrededor de la estación meteorológica en consideración y la posición aproximada señalada de descarga eléctrica atmosférica.

$\mathrm{Se}$ formaron agrupaciones de diferencia de temperatura de brillo teniendo en consideración los siguientes criterios de asociación y cumplimiento:

a. Los valores de diferencia de temperatura de brillo pertenecerán a un mismo agrupamiento cuando la distancia de separación alrededor de la estación meteorológica sea menor a $10 \mathrm{~km}$.

b. Los valores de diferencia de temperatura de brillo pertenecerán a un mismo agrupamiento cuando las descargas ocurren consecutivamente en un lapso de tiempo menor a 20 minutos.

c. Los valores de diferencia de temperatura de brillo pertenecerán a un mismo agrupamiento cuando el valor absoluto de estas diferencias sea menor a $3.2^{\circ} \mathrm{C}$, siendo este valor el obtenido después del tratamiento estadístico aplicado a los datos buscando los anchos de clases.

$\underline{\text { Relaciones empíricas y de probabilidades de descargas }}$ eléctricas atmosféricas en base a la diferencia de temperatura de brillo

Se realizó el análisis exploratorio de datos (AED) a los archivos en formato de hoja de cálculo obtenidos de la base de datos de descargas eléctricas atmosféricas del STARNET y archivos NetCDF de imágenes de satélite GOES 13 en sus bandas 3 y 4, eliminándose aquellas filas y columnas que contuvieron datos faltantes y/o atípicos. 
Ajuste del modelo de regresión lineal

De las agrupaciones de diferencia de temperatura de brillo encontradas y mediante el software $\mathrm{R}$, se descompuso la variable TB3 - TB4 en su mínimo, máximo, media y mediana promedio por hora, día y mes, como variables predictoras.

Se utilizó un modelo de regresión lineal al momento de predecir los valores de ocurrencia de descargas eléctricas atmosféricas a partir de los valores de diferencia de temperatura de brillo entre la banda 3 y banda 4 del GOES 13 (modelo de regresión lineal múltiple) obteniéndose un modelo del tipo de la Fórmula 1.

$$
\hat{Y}=\beta_{0}+\beta_{1} X_{1}+\beta_{2} X_{2}+\beta_{3} X_{3}
$$

Fórmula 1.

Donde:

$\mathrm{X}_{1}$ : Diferencia mínima de temperatura de brillo.

$\mathrm{X}_{2}$ : Diferencia máxima de temperatura de brillo.

$\mathrm{X}_{3}$ : Diferencia promedio de temperatura de brillo.

$\mathrm{Al}$ momento de realizar las predicciones es probable que se hayan cometido errores de predicción, calculadas con la Fórmula 2.

$$
E_{i}=Y_{i}-\hat{Y}_{i}
$$

\section{Fórmula 2.}

Donde:

$\mathrm{Y}_{\mathrm{i}}$ : Valor observado

$\hat{Y}_{\mathrm{i}}$ : Valor estimado

Un criterio natural de la prueba de bondad de ajuste fue considerar la ecuación de regresión que proporcione un menor error en las predicciones, lo que permite tener un mejor ajuste con la ecuación de regresión cuyo error haya estado más próximo al valor cero.

Se ajustó el modelo de regresión lineal con los datos a disposición para predecir el número de descargas promedio por caso (nube) y por hora; para la comunidad campesina de Marcapomacocha se utilizó la totalidad de los datos recopilados mientras que para la comunidad campesina de Huayao solo se trabajó con los datos del año 2016 por ser el año en la que se tienen registros más completos en relación a los otros años considerados para el estudio.

Relaciones de probabilidades

Los datos se ordenaron en función al tiempo de ocurrencia de descargas eléctricas, desde el más antiguo hasta el más reciente, generándose cuatro nuevos archivos. El primero se utilizó para observar al detalle, la ubicación y la cantidad de descargas eléctricas pertenecientes a un solo sistema convectivo y sus características referidas a la temperatura de brillo de las bandas de vapor de agua e infrarroja, además de la diferencia de temperatura de brillo entre estas bandas. El segundo se utilizó para tener un conteo más detallado de las descargas eléctricas y su relación con los sistemas convectivos mediante el valor mínimo, máximo y promedio de diferencia de temperatura de brillo entre la banda 3 y banda 4 .

En el tercero se encuentran los arreglos del número de rayos por hora. De manera análoga sucede con el cuarto y quinto archivo, que consideran dichas características por día y por mes, respectivamente.

En cuanto al análisis inferencial, se plantearon pruebas de hipótesis con un nivel de significancia de 0.05 , para verificar la dependencia entre el número de descargas y el tiempo (hora, día, mes, año) con la cantidad de descargas por sistema convectivo. La relación con la que se calculan las probabilidades de ocurrencia de rayos tiene la forma de la Fórmula 3.

$\operatorname{Prob}\left(\mathrm{N}_{\text {descargas }}=\mathrm{y}\right)=\mathrm{e}^{\left(\beta_{0}+\beta_{1} \text { DIF_MAX }_{-} \beta_{2} \mathrm{DIF}_{-} \mathrm{MIN}+\beta_{3} \text { DIF_PROM }\right)}$

\section{Fórmula 3.}

Donde:

Y: Número de descargas o rayos previsto.

$\beta_{0}$ : Intercepto.

$\beta_{1}$ : Coeficiente de la variable diferencia de temperatura máxima.

$\beta_{2}$ : Coeficiente de la variable diferencia de temperatura mínima.

$\beta_{3}$ : Coeficiente de la variable diferencia de temperatura promedio.

Diagnóstico de descargas eléctricas atmosféricas

El meteorólogo especialista del Servicio Meteorológico a través de la técnica de pronóstico de corto plazo, identifica la ubicación de las localidades que pueden ser afectadas por tormentas eléctricas y realiza el monitoreo continuo de las formaciones nubosas con potencial para ocurrencia de descargas eléctricas de origen atmosférico; determinando la evolución de las temperaturas de brillo para las bandas de vapor de agua e infrarrojo.

\section{Resultados y discusión}

Georreferenciación de puntos de descargas eléctricas

Para las estaciones meteorológicas de Marcapomacocha y Huayao se georreferenciaron 911 y 189 descargas eléctricas respectivamente, que corresponden a la ocurrencia entre junio del 2015 a noviembre del 2017 (Tabla 2).

Diferencia de temperatura de brillo entre la banda de vapor de agua e infrarrojo

Se observó la frecuencia absoluta de descargas eléctricas atmosféricas sobre la comunidad campesina de Marcapomacocha (Figura 2), con un comportamiento que se aproxima a una distribución Fisher-Snedecor, pero sin suficiente significancia estadística.

Para la comunidad campesina de Huayao (Figura 3), que tiene menor cantidad de datos respecto de Marcapomacocha, la distribución de la frecuencia 
Tabla 2. Georreferenciación de puntos de descargas eléctricas atmosféricas.

\begin{tabular}{crrrrrrc}
\hline Año & Mes & Día & Hora & Minuto & Segundo & Latitud Sur $\left({ }^{\circ}\right)$ & Longitud Oeste $\left(^{\circ}\right)$ \\
\hline 2016 & 10 & 1 & 12 & 50 & 7 & -10.343580 & -75.040510 \\
2016 & 10 & 1 & 12 & 54 & 45 & -10.566250 & -75.004450 \\
2016 & 10 & 1 & 12 & 59 & 16 & -10.439140 & -75.085300 \\
2016 & 10 & 1 & 12 & 59 & 16 & -10.434270 & -75.052310 \\
2016 & 10 & 1 & 12 & 59 & 16 & -10.443020 & -75.049510 \\
2016 & 10 & 1 & 12 & 59 & 16 & -10.447570 & -75.063010 \\
2016 & 10 & 1 & 13 & 12 & 50 & -10.441550 & -75.047000 \\
2016 & 10 & 1 & 13 & 16 & 22 & -10.382940 & -75.239650 \\
2016 & 10 & 1 & 13 & 17 & 13 & -10.322110 & -75.292950 \\
2016 & 10 & 1 & 13 & 17 & 6 & -10.447310 & -75.084690 \\
2016 & 10 & 1 & 13 & 20 & 21 & -10.050710 & -75.092070 \\
2016 & 10 & 1 & 13 & 32 & 4 & -10.432840 & -75.225140 \\
\hline
\end{tabular}

absoluta también presenta una aproximación a una distribución Fisher-Snedecor, pero sin suficiente significancia estadística.

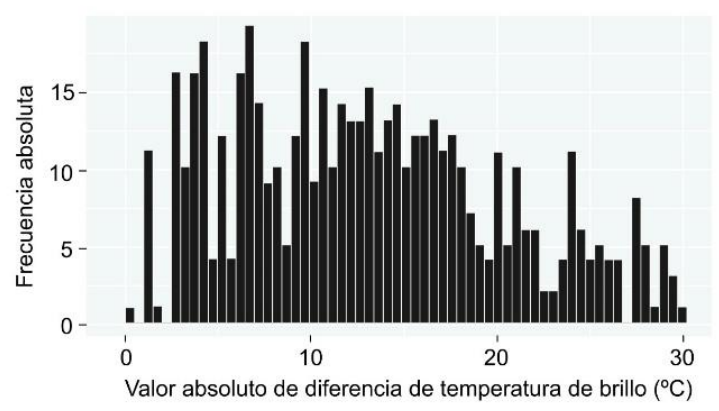

Figura 2. Frecuencia absoluta de descargas eléctricas de origen atmosférico en la comunidad campesina de Marcapomacocha.

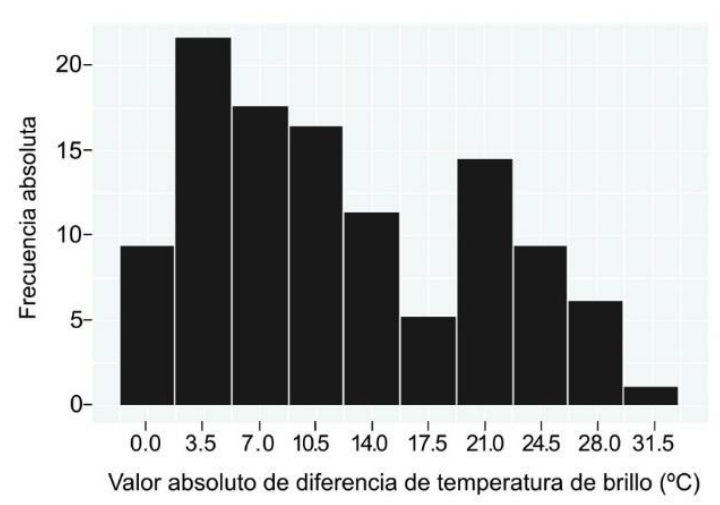

Figura 3. Frecuencia absoluta de descargas eléctricas de origen atmosférico en la comunidad campesina de Huayao.

Relaciones empíricas y de probabilidades entre la ocurrencia de descargas eléctricas y diferencia de temperatura de brillo

El modelo multinomial utilizado, calcula las probabilidades de pertenecer a cierta categoría. Las categorías para el caso de Marcapomacocha, son los números de descargas, es decir para 1, 2, 3, 4, 5, 6, 8, 10 y 12 rayos.
$\mathrm{P} 2=\mathrm{e}^{(-2.695+45.6 \text { DIF_MAX-10.2DIF_MIN-35.5DIF_PROM })}$ $\mathrm{P} 3=\mathrm{e}^{(-4.68+46.1 \mathrm{DIF} \text { MAX }-10.2 \mathrm{DIF} \text {-MIN- 35.9DIF_PROM })}$

P4 $=\mathrm{e}^{\left(-5.71+45.3 \text { DIF_MAX }-7.7 \text { DIF_MIN }-37.8 \text { DIF_PROM }_{-}\right)}$

$\mathrm{P} 5=\mathrm{e}^{\left(-6.18+45.0 \mathrm{DIF} \text { MAX }-7.3 \mathrm{DIF} \_\mathrm{MIN}-37.8 \mathrm{DIF} \text { _PROM }\right)}$

$\mathrm{P} 6=\mathrm{e}^{(-4.46+51.2 \text { DIF_MAX-20.2DIF_MIN-31.4DIF_PROM })^{-}}$

$\mathrm{P} 8=\mathrm{e}^{(-68.1+38.3 \text { DIF_MAX }+24.5 \text { DIF_MIN-62.2DIF_PROM })}$

P10 $=\mathrm{e}^{(-6.66+48.8 \text { DIF_MAX-15.3DIF_MIN-33.6DIF_PROM })_{-}}$

$\mathrm{P} 12=\mathrm{e}^{(-9.21-52.6 \text { DIF_MAX-23.4DIF_MIN-29.3DIF_PROM })}$

$\mathrm{P} 1=1-(\mathrm{P} 2+\mathrm{P} 3+\mathrm{P} 4+\mathrm{P} 5+\mathrm{P} 6+\mathrm{P} 8+\mathrm{P} 10+\mathrm{P} 12)$

Para Huayao, el modelo multinomial utilizado, calcula las probabilidades de pertenecer a cierta categoría. Para este caso son los números de descargas para $1,2,3,5,6$ y 9 rayos.

$\mathrm{P} 2=\mathrm{e}^{\left(-2.857+187.8 D I F_{-} M A X+20.06 \text { DIF_MIN-207.3DIF_PROM) }\right.}$ $\mathrm{P} 3=\mathrm{e}^{\left(-3.287+168.4 \mathrm{DIF}_{-} \mathrm{MAX}+53.73 \mathrm{DIF} \text { MIN-222.1DIF_PROM }\right)}$

$\mathrm{P} 5=\mathrm{e}^{\left(8.773+68.02 \mathrm{DIF}_{\mathrm{MAX}}+218.8 \mathrm{DIF}_{\mathrm{MIN}}-286.99 \text { DIF_PROM }_{\text {_ }}\right.}$

$\mathrm{P} 6=\mathrm{e}^{(-15.66+263 \mathrm{DIF} \text { _MAX-141.7DIF_MIN-121.23DIF_PROM })}$

$\mathrm{P} 9=\mathrm{e}^{\left(-106.5+322 \mathrm{DIF}_{-} \mathrm{MAX}-149.2 \mathrm{DIF}_{-} \mathrm{MIN}-172.13 \mathrm{DIF}_{-} \text {PROM }\right)}$

$$
P 1=1-(P 2+P 3+P 5+P 6+P 9)
$$

Diagnóstico de la ocurrencia de descargas eléctricas atmosféricas sobre la cuenca del río Mantaro

PRONÓSTICO DE CORTO PLAZO

Durante las pruebas, los pronosticadores mediante la técnica de pronósticos de corto plazo se encargaron de hacer seguimiento a los sistemas nubosos que puedan generar descargas eléctricas, para tal efecto hicieron uso de imágenes de satélite en las bandas ya señaladas, centrándose en las comunidades campesinas de Marcapomacocha y Huayao durante los días 8 y 9 de marzo del 2019 respectivamente. La variación temporal de la temperatura de brillo para la banda infrarroja sobre las localidades de Marcapomacocha y Huayao fue monitoreado mediante la herramienta "Puntograma" (Figuras 4 y 5, respectivamente), observándose en ambos casos la disminución inesperada de temperatura de brillo, indicador de un crecimiento continuo de la nubosidad.

Comprobación de la utilización de diferencias de temperatura de brillo en los modelos de pronósticos y cálculo de probabilidades de ocurrencia de rayos

Con la información del satélite GOES 13, para el cálculo de la tasa de aciertos en Marcapomacocha, se utilizaron los datos del periodo 2015-2016 para 
entrenar el modelo y los datos del año 2017 y hallar la tasa de aciertos; para tal fin, se hizo uso del software "Rstudio", llegándose a obtener aciertos de hasta $88 \%$ (Fórmula 4).

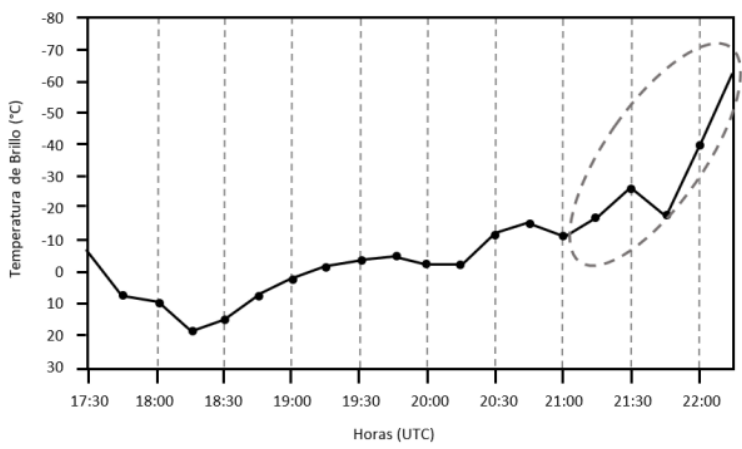

Figura 4. Puntograma para verificación de temperatura de brillo de la banda 14 del GOES 16 para la zona de Marcapomacocha (08 de marzo de 2019).

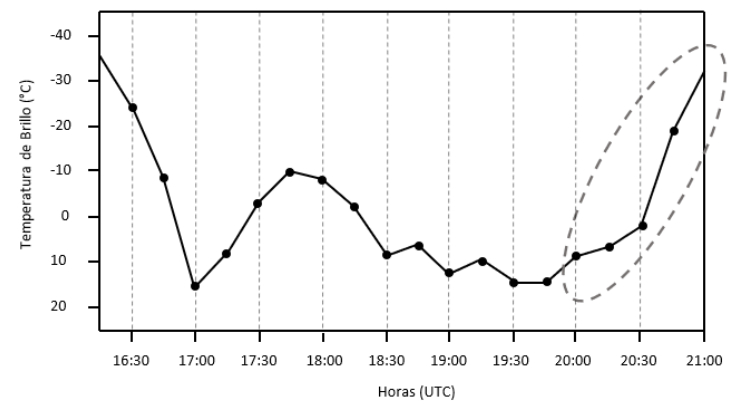

Figura 5. Puntograma para verificación de temperatura de brillo de la banda 14 del GOES 16 para la zona de Huayao (09 de marzo de 2019).

>\#\#\#\#\#\#\#\#\#\#\#\#\#\# validación con los datos del año 2017

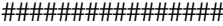

$>$ pred $=$ predict $(\bmod 1$, test,type="class" $)$

$>$

$>$ \#\#\# TASA DE ACIERTO \#\#\#\#

$>$ sum(diag(as.matrix(table(test\$DESCARGAS,pred)) [1:8,1:8])

)/sum(as.matrix(table(test\$DESCARGAS,pred)[1:8,1:8]))

[1] 0.8790323

\section{Fórmula 4.}

De manera similar para Huayao, se separaron los datos de 2016 para entrenar el modelo y los datos de los años 2015 y 2017 para hallar su tasa de aciertos, llegándose a obtener aciertos de hasta 100\% (Fórmula 5).

Comprobación utilizando banda 9 y banda 14 del satélite GOES 16

Las comprobaciones se llevaron a cabo utilizando los archivos NetCDF diarios de la banda 9 y banda 14 del GOES 16 que son equivalentes a la banda 3 y banda 4 del GOES 13 respectivamente, extrayéndose los respectivos valores de temperatura de brillo para el periodo de tiempo de las simulaciones y localizaciones dentro de un radio de $10 \mathrm{~km}$ alrededor de cada comunidad campesina en estudio, aplicando los algoritmos respectivos (Tabla 3).

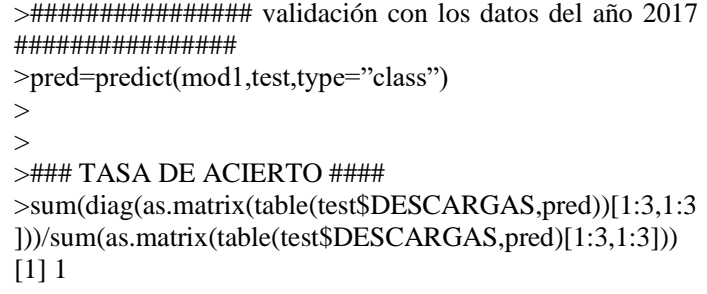

\section{Fórmula 5.}

Podemos observar que para la comunidad campesina de Marcapomacocha, la ocurrencia de descargas eléctricas atmosféricas tiene lugar a partir de la diferencia de temperatura de brillo de $-4{ }^{\circ} \mathrm{C}$ mientras que para Huayao es a partir de $-7^{\circ} \mathrm{C}$.

Tabla 3. Ocurrencia de descargas eléctricas atmosféricas en las comunidades campesinas focales de Marcapomacocha y Huayao.

\begin{tabular}{ccc}
\hline Diferencia de & \multicolumn{2}{c}{ Estación meteorológica } \\
\cline { 2 - 3 } $\begin{array}{c}\text { Temperatura de } \\
\text { Brillo }\end{array}$ & Marcapomacocha & Huayao \\
(TB9- TB14) ${ }^{\circ} \mathbf{C}$ & $\mathrm{SD}$ & $\mathrm{SD}$ \\
\hline-1 & $\mathrm{SD}$ & $\mathrm{SD}$ \\
-2 & $\mathrm{SD}$ & $\mathrm{SD}$ \\
-3 & $\mathrm{Si}$ & $\mathrm{No}$ \\
-4 & $\mathrm{Si}$ & $\mathrm{No}$ \\
-5 & $\mathrm{Si}$ & $\mathrm{No}$ \\
-6 & $\mathrm{Si}$ & $\mathrm{Si}$ \\
-7 & $\mathrm{Si}$ & $\mathrm{Si}$ \\
-8 & $\mathrm{Si}$ & $\mathrm{Si}$ \\
-9 & $\mathrm{Si}$ & $\mathrm{Si}$ \\
-10 & $\mathrm{Si}$ & $\mathrm{Si}$ \\
-11 & $\mathrm{Si}$ & $\mathrm{Si}$ \\
-12 & $\mathrm{Si}$ & $\mathrm{Si}$ \\
-13 & $\mathrm{SO}$ & $\mathrm{Na}$
\end{tabular}

SI: Si hay ocurrencia de rayos. NO: No hay ocurrencia de rayos. SD: Sin datos.

Evolución de la diferencia de temperatura de brillo (TB9-TB14) en otras estaciones de la cuenca del río Mantaro durante simulaciones

Durante las pruebas, se pudo comprobar que, el día 8 de marzo en las estaciones de La Oroya y Tarma (Figura 6; $f$ y $h$, respectivamente) se presentaron pocas variaciones de diferencias de temperatura de brillo entre los casos de descargas eléctricas acontecidas; mientras que, en las estaciones Yauli, Santa Ana y Carhuamayo (Figura 6; $a, g$ y $c$, respectivamente) las diferencias de temperatura de brillo entre los casos de descargas presentados muestran amplias variaciones; para el día 9 de marzo en las estaciones de Carhuamayo y Yantac (Figura 7; $c$ e $i$, respectivamente) se presentaron pocas variaciones de diferencias de temperatura de brillo entre los diferentes casos de descargas eléctricas acontecidas; mientras que, en las 
Tabla 4. Ocurrencia de descargas eléctricas atmosféricas en otras comunidades campesinas.

\begin{tabular}{|c|c|c|c|c|c|c|c|c|c|c|}
\hline \multirow[b]{2}{*}{$\begin{array}{c}\text { Diferencia de } \\
\text { Temperatura } \\
\text { de Brillo } \\
\text { (TB9-TB14) } \\
{ }^{\circ} \mathrm{C}\end{array}$} & \multicolumn{10}{|c|}{ Estación meteorológica } \\
\hline & 焉 & 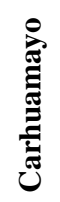 & 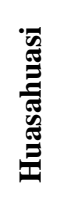 & 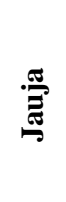 & 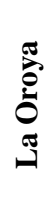 & 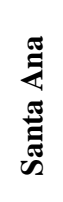 & 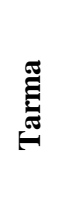 & $\stackrel{\mathscr{\theta}}{\vec{E}}$ & 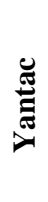 & : \\
\hline-1 & No & No & No & No & No & No & No & No & $\mathrm{Si}$ & No \\
\hline-2 & $\mathrm{Si}$ & No & No & $\mathrm{Si}$ & No & $\mathrm{Si}$ & No & No & $\mathrm{Si}$ & No \\
\hline-3 & $\mathrm{Si}$ & $\mathrm{Si}$ & $\mathrm{Si}$ & $\mathrm{Si}$ & $\mathrm{Si}$ & $\mathrm{Si}$ & $\mathrm{Si}$ & $\mathrm{Si}$ & $\mathrm{Si}$ & $\mathrm{Si}$ \\
\hline-4 & $\mathrm{Si}$ & $\mathrm{Si}$ & $\mathrm{Si}$ & $\mathrm{Si}$ & $\mathrm{Si}$ & $\mathrm{Si}$ & $\mathrm{Si}$ & $\mathrm{Si}$ & $\mathrm{Si}$ & $\mathrm{Si}$ \\
\hline-5 & $\mathrm{Si}$ & $\mathrm{Si}$ & $\mathrm{Si}$ & $\mathrm{Si}$ & $\mathrm{Si}$ & $\mathrm{Si}$ & $\mathrm{Si}$ & $\mathrm{Si}$ & $\mathrm{Si}$ & $\mathrm{Si}$ \\
\hline-6 & $\mathrm{Si}$ & $\mathrm{Si}$ & $\mathrm{Si}$ & $\mathrm{Si}$ & $\mathrm{Si}$ & $\mathrm{Si}$ & $\mathrm{Si}$ & $\mathrm{Si}$ & $\mathrm{Si}$ & $\mathrm{Si}$ \\
\hline-7 & $\mathrm{Si}$ & $\mathrm{Si}$ & $\mathrm{Si}$ & $\mathrm{Si}$ & $\mathrm{Si}$ & $\mathrm{Si}$ & $\mathrm{Si}$ & $\mathrm{Si}$ & $\mathrm{Si}$ & $\mathrm{Si}$ \\
\hline-8 & $\mathrm{Si}$ & $\mathrm{Si}$ & $\mathrm{Si}$ & $\mathrm{Si}$ & $\mathrm{Si}$ & $\mathrm{Si}$ & $\mathrm{Si}$ & $\mathrm{Si}$ & $\mathrm{Si}$ & $\mathrm{Si}$ \\
\hline-9 & $\mathrm{Si}$ & $\mathrm{Si}$ & $\mathrm{Si}$ & $\mathrm{Si}$ & $\mathrm{Si}$ & $\mathrm{Si}$ & $\mathrm{Si}$ & $\mathrm{Si}$ & $\mathrm{Si}$ & $\mathrm{Si}$ \\
\hline-10 & $\mathrm{Si}$ & $\mathrm{Si}$ & $\mathrm{Si}$ & $\mathrm{Si}$ & $\mathrm{Si}$ & $\mathrm{Si}$ & $\mathrm{Si}$ & $\mathrm{Si}$ & $\mathrm{Si}$ & $\mathrm{Si}$ \\
\hline-11 & $\mathrm{Si}$ & $\mathrm{Si}$ & $\mathrm{Si}$ & $\mathrm{Si}$ & $\mathrm{Si}$ & $\mathrm{Si}$ & $\mathrm{Si}$ & $\mathrm{Si}$ & $\mathrm{Si}$ & $\mathrm{Si}$ \\
\hline-12 & $\mathrm{Si}$ & $\mathrm{Si}$ & $\mathrm{Si}$ & $\mathrm{Si}$ & $\mathrm{Si}$ & $\mathrm{Si}$ & $\mathrm{Si}$ & $\mathrm{Si}$ & $\mathrm{Si}$ & $\mathrm{Si}$ \\
\hline-13 & $\mathrm{Si}$ & $\mathrm{Si}$ & $\mathrm{Si}$ & $\mathrm{Si}$ & $\mathrm{Si}$ & $\mathrm{Si}$ & $\mathrm{Si}$ & $\mathrm{Si}$ & $\mathrm{Si}$ & $\mathrm{Si}$ \\
\hline
\end{tabular}

SI: Si hay ocurrencia de rayos. NO: No hay ocurrencia de rayos. SD: Sin datos.

estaciones Jauja y Yauli (Figura 7; j y $a$, respectivamente) las variaciones de diferencias de temperatura de brillo tienen mayor amplitud; situaciones que sugieren que en los casos donde hay poca variación, las nubosidades presentadas que generan descargas eléctricas se muestran de manera uniforme y posiblemente formando conglomerados compactos, y en los casos donde hay amplias variaciones, las nubosidades que los generan son muy irregulares o en su defecto de algún modo dispersos.

Podemos observar en la Tabla 4 , un resumen de la ocurrencia de descargas eléctricas atmosféricas en otras comunidades campesinas, entre los días 8 y 9 de marzo del 2019, en la misma podemos observar que, la ocurrencia de descargas eléctricas atmosféricas tiene lugar a partir de la diferencia de temperatura de brillo entre $-1^{\circ} \mathrm{C}$ y $-2^{\circ} \mathrm{C}$.

\section{Conclusiones}

El número de descargas varía en función de los meses del año siendo esta una condición relevante para el cálculo de probabilidades del número de descargas por caso (nube) asociadas a un nivel de significancia de 0.05; las relaciones empíricas y de probabilidades encontradas en este estudio permitieron estimar la ocurrencia de descargas eléctricas atmosféricas en las localidades de Huayao y Marcapomacocha.

Mediante esta novedosa técnica, se obtendrían mejores asociaciones entre la ocurrencia de descargas eléctricas atmosféricas con la temperatura de brillo de las bandas adecuadas proveniente de los satélites meteorológico, para este efecto se debería utilizar mayor cantidad de datos para obtener una relación óptima entre ambos sistemas.
Los resultados sugieren que, realizando los ajustes necesarios de precisión en la detección de rayos, se podría diseñar e implementar un óptimo sistema de monitoreo de ocurrencia de descargas eléctricas atmosféricas para cualquier zona del país; para este efecto se propone encontrar umbrales de diferencia de temperatura de brillo para que haya actividad eléctrica, utilizando información de redes terrestres y datos de satélites meteorológicos, entre otros.

\section{Agradecimientos}

Se agradece a la Dra. Elba Fiallio-Pantziou del Centro Internacional de Investigación del Fenómeno El Niño, al Dr. Carlos Augusto Morales de la Universidad de Sao Paulo por facilitar la información referente a las descargas eléctricas de origen atmosférico; a la Ing. Bremilda Sutizal, al Ing. Benito Valderrama y al Bach. Cristian Dávila del Servicio Nacional de Meteorología e Hidrología del Perú por el apoyo antes y durante los trabajos de campo. A la Dra. Yamina Silva del Instituto Geofisico del Perú por proporcionar información referida a la Cuenca del río Mantaro; a Yajaira Figueroa, Ángel Maco, Erick Alvarez y Diego Díaz, jóvenes estudiantes de la Universidad Nacional Agraria La Molina que apoyaron incansablemente en la elaboración del estudio y agradecimiento especial al Sr. Luis Antezano y Sra. Marcelina Melgar de la comunidad campesina de Huayao y a la Sra. Julia Astudillo de la comunidad campesina de Marcapomacocha. 


\section{Literatura citada}

Abdoulaev S., Marques V.S., Pinheiro F.M.A., Martinez E.F.A. \& Lenskaia O. 2001. Analysis of mesoscale system using-to-ground flash data. Braz. J. Geophys, 19(1): $\quad 75-95 . \quad$ DOI: $\quad 10.1590 /$ S0102261X2001000100006.

Arellano L.F. \& Escalante S.C. 2014. Estimación del potencial de tormentas vía la combinación de imágenes satelitales e información meteorológica: caso de estudio al noroeste de México. Tecnología y Ciencias del Agua, 5(5):

39-61. http://www.scielo.org.mx/scielo.php?script=sci_abstract \&pid=S2007-24222014000500003\&lng=es\&nrm=iso.

Baker M.B., Christian H.J. \& Latham J. 1995. A computational study of the relationships linking lightning frequency and other thundercloud parameters. Q. J. R. Meteorol. Soc., 121: 1525-1548. https://www.academia.edu/22402807/A_computational_ study_of_the_relationships_linking_lightning_frequency _and_other_thundercloud_parameters.

Deierling W. \& Petersen W.A. 2008. Total lightning activity as an indicator of updraft characteristics. Journal of Geophysical Research: Atmospheres, 113, D16210: 1 11. DOI: $10.1029 / 2007$ JD009598.

Falcón N. \& Quintero A. 2009. Electrometeoros y aerosoles piroeléctricos en tormentas tropicales. Revista Ingeniería, 16(3): 27-33. http://servicio.bc.uc.edu.ve/ingenieria/revista/a16n3/art4 .pdf.

Herzegh P.H. \& Jameson A.R. 1992. Observing precipitation through dual polarization radar measurements. Bulletin of the American Meteorological Society, 73(9): 1365-1376. DOI:

$10.1175 / 1520$ 0477(1992)073<1365:OPTDPR>2.0.CO;2.

Homeyer C.R. \& Kumjian M.R. 2015. Microphysical characteristics of overshooting convection from polarimetric radar observations. Journal of the Atmospheric Sciences, 72(2): 870-891. DOI: 10.1175/JAS-D-13-0388.1.

Keith W. \& Saunders C. 1990. Further laboratory studies of the charging of graupel during ice crystal interactions. Atmospheric Research, 25(5): 445-464. DOI: 10.1016/0169-8095(90)90028-B.

Kumjian M.R., Khain A.P., Benmoshe N., Ilotiviz E., Ryzhkov A.V. \& Phillips V.T. 2014. The anatomy and physics of zdr columns: investigating a polarimetric radar signature with a spectral bin microphysical model. Journal of Applied Meteorology and Climatology, 53(7): 1820-1843. DOI: 10.1175/JAMC-D-13-0354.1.

Lang T.J. \& Rutledge S.A. 2002. Relationships between convective storm kinematics, precipitation, and lightning. Monthly Weather Review, 130(10): 2492-2506. DOI: $10.1175 / 1520$ 0493(2002)130<2492:RBCSKP>2.0.CO;2.

Lee A.C.L. 1986. An operational system for remote location of lightning flashes using a VLF arrival time difference technique. J. Atmos. and Ocean. Tech., 3(4): 630-642. https://doi.org/10.1175/15200426(1986)003\%3C0630:AOSFTR\%3E2.0.CO;2.
Machado L., Lima W., Pinto O. (Jr.) \& Morales C. 2009. Relationship between cloud-ground Lightning and Penetrative Clouds: A Multi-channel Satellite Application, 93: 304-309. DOI: 10.1016/j.atmosres.2008.10.003

Mattos E.V., Machado L.A., Williams E.R., Goodman S.J., Blakeslee R.J. \& Bailey J.C. 2017. Electrification life cycle of incipient thunderstorms. Journal of Geophysical Research: Atmospheres, 122(8): 4670-4697. DOI: 10.1002/2016JD025772.

Mecikalski J.R. \& Bedka K.M. 2006. Forecasting convective initiation by monitoring the evolution of moving cumulus in daytime goes imagery. Monthly Weather Review, 134(1): 49-78. DOI: 10.1175/MWR3062.1.

Mecikalski J.R., Bedka K.M., Paech S.J. \& Litten L.A. 2008. A statistical evaluation of goes cloud-top properties for nowcasting convective initiation. Monthly Weather Review, 136(12): 4899-4914. DOI: 10.1175/2008MWR2352.1.

WMO (WORLD METEOROLOGICAL ORGANIZATION). 2017. Thunderstorm (Section 3.2.4.1). In: WMO International Cloud Atlas. Manual on the Observation of Clouds and Other Meteors (WMO-No. 407). 2017 Edition. Consultado entre el 09 y 15 de febrero de 2021 de: https://cloudatlas.wmo.int/es/thunderstorm.html.

WMO (WORLD METEOROLOGICAL ORGANIZATION). 2020. WMO certifies Megaflash lightning extremes. In: Press Release [of WMO], Published 24 June 2020 Number: 26062020. Consultado entre el 09 y 15 de febrero de 2021 de: https://public.wmo.int/en/media/press-release/wmocertifies-megaflash-lightning-extremes.

Petersen W.A. \& Rutledge S.A. 1998. On the relationship between cloud-toground lightning and convective rainfall. Journal of Geophysical Research: Atmospheres, 103(D12): 14025-14040. DOI: 10.1029/97JD02064.

Saunders C.P.R., Keith W.D. \& Mitzeva R.P. 1991. The effect of liquid water on thunderstorm charging. Journal Geophys. Res., 96(11): 007-017. DOI: 10.1029/91JD00970.

Snyder J.C., Ryzhkov A.V., Kumjian M.R., Khain A.P. \& Picca J. 2015. A $\mathrm{z}$ dr column detection algorithm to examine convective storm updrafts. Weather and Forecasting, 30(6): 1819-1844. DOI: 10.1175/WAF-D15-0068.1.

STARNET (Sferics Timing and Ranging Network). 2017. http://www.zeus.iag.usp.br/ftp/. Consultado entre junio de 2015 y noviembre de 2017.

Tessendorf S.A., Miller L.J., Wiens K.C. \& Rutledge S.A. 2005. The 29 June 2000 supercell observed during steps. part i: kinematics and microphysics. Journal of the Atmospheric Sciences, 62(12): 4127-150. DOI: 10.1175/JAS3585.1.

Vila D.A., Machado L.A.T., Laurent H. \& Velasco I. 2008. Forecast and Tracking the Evolution of Cloud Clusters (ForTraCC) Using Satellite Infrared Imagery: Methodology and Validation. Weather and Forecasting, 23(2): 233-245. American Meteorological Society. DOI: 10.1175/2007WAF2006121.1.

Wallace J.M. \& Hobbs P.V. 2006. Atmospheric Science an Introductory Survey. 2 ed. University Washington. 


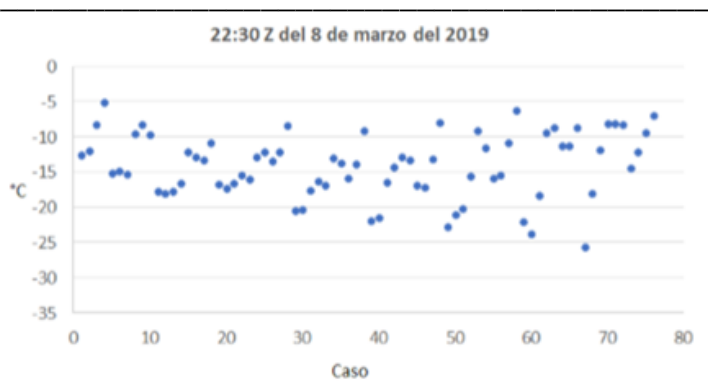

a. Estación: Yauli

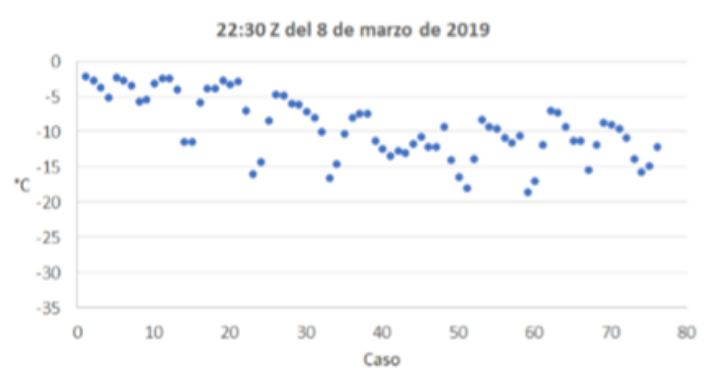

b. Estación: Carhuacayán

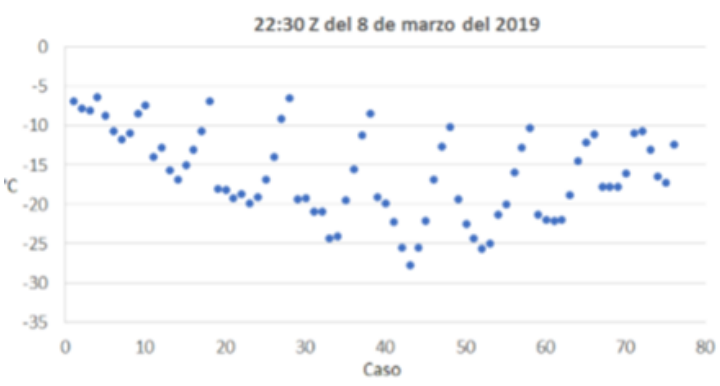

c. Estación: Carhuamayo

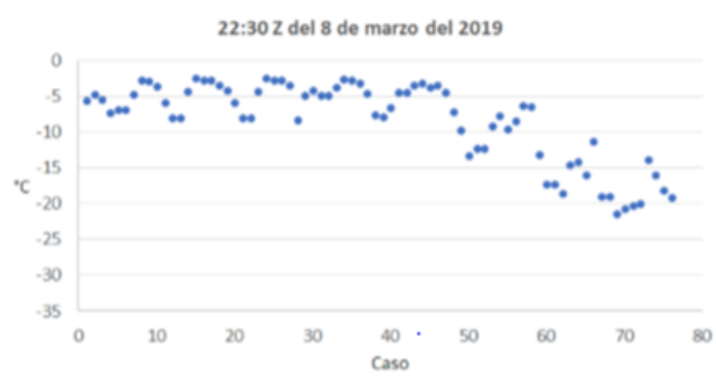

d. Estación: Huasahuasi

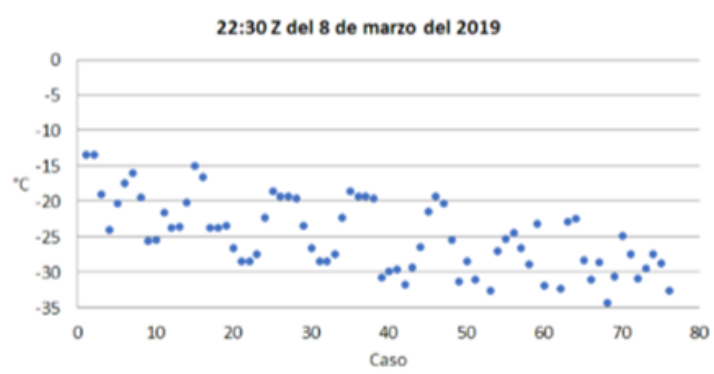

e. Estación: Viques

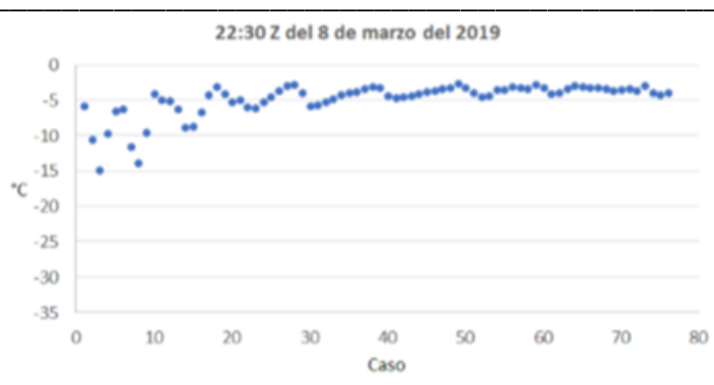

f. Estación: La Oroya

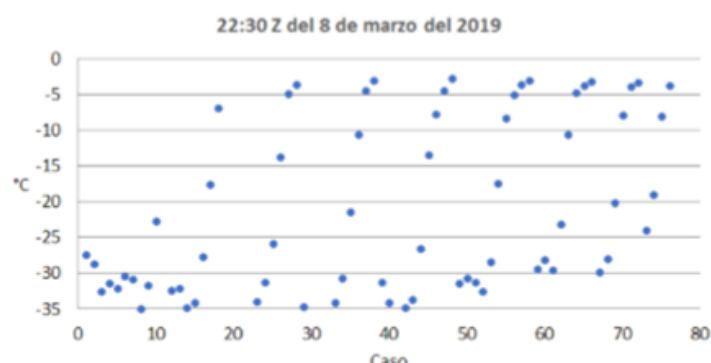

g. Estación: Santa Ana

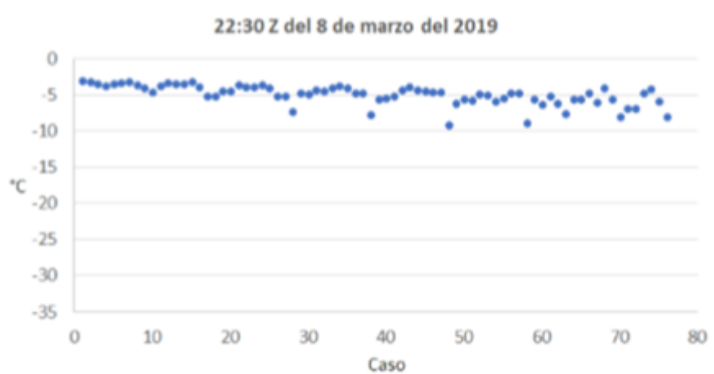

h. Estación: Tarma

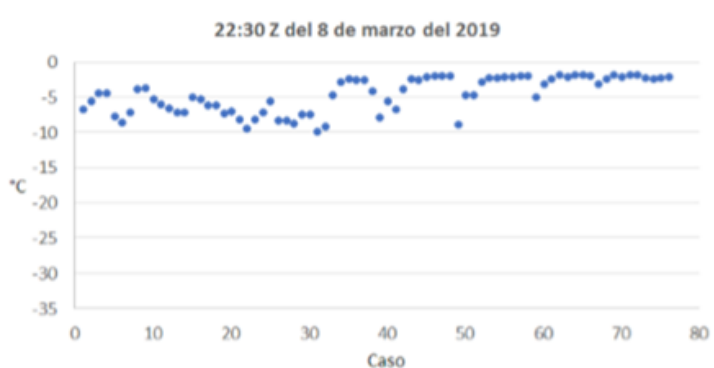

i. Estación: Yantac

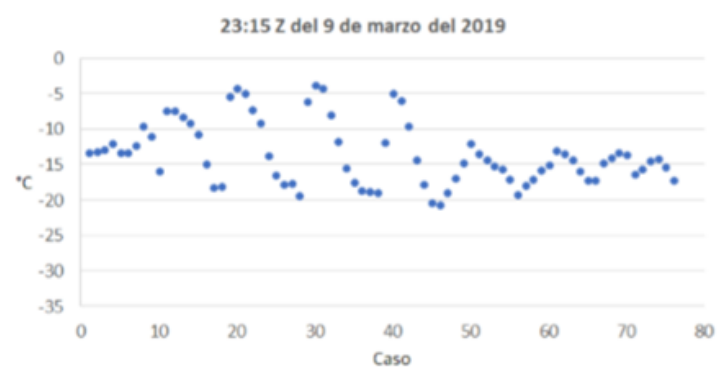

j. Estación: Jauja

Figura 6. Diferencia de temperatura de brillo (TB9-TB14) del satélite GOES 16 en las estaciones de la cuenca del río Mantaro durante las simulaciones. 08 de marzo del 2019. 


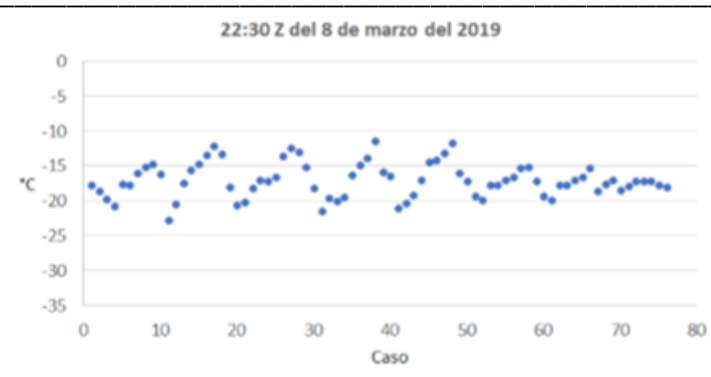

a. Estación: Yauli

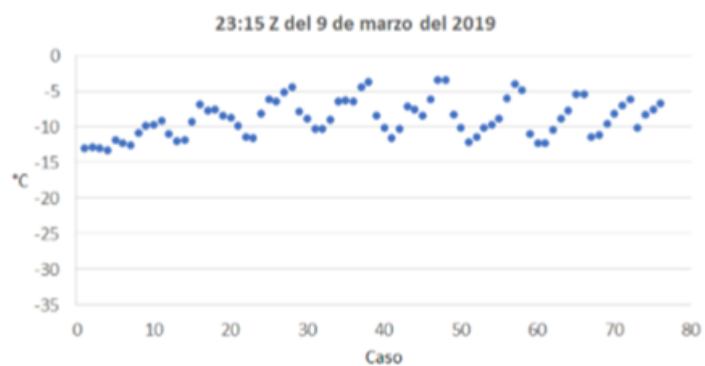

b Estación: Carhuacayán

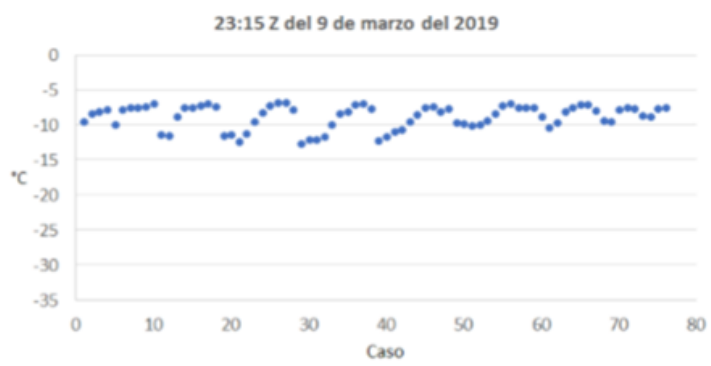

c. Estación: Carhuamayo

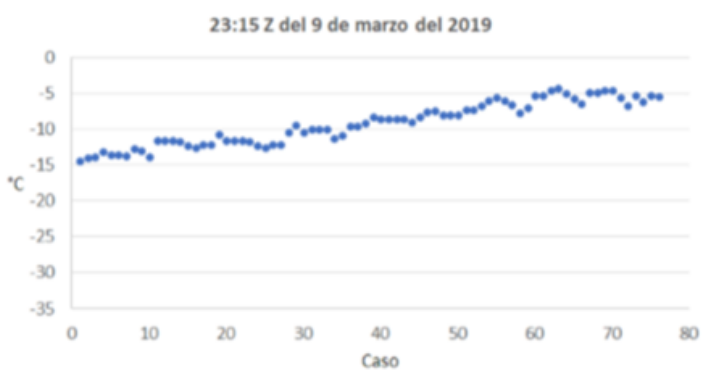

d. Estación: Huasahuasi

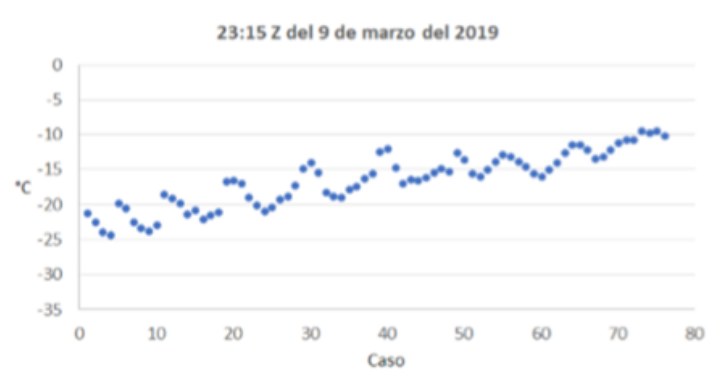

e. Estación: Viques

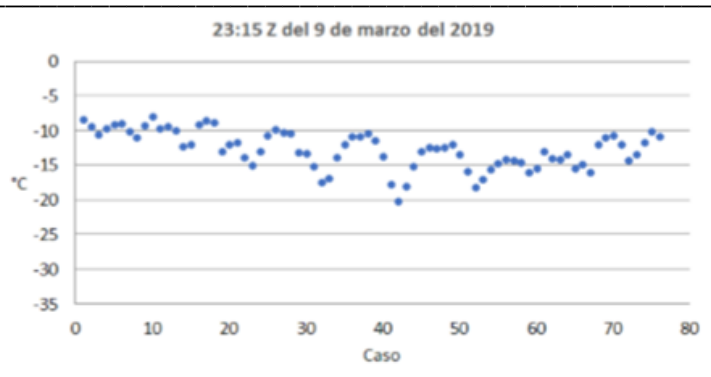

f. Estación: La Oroya

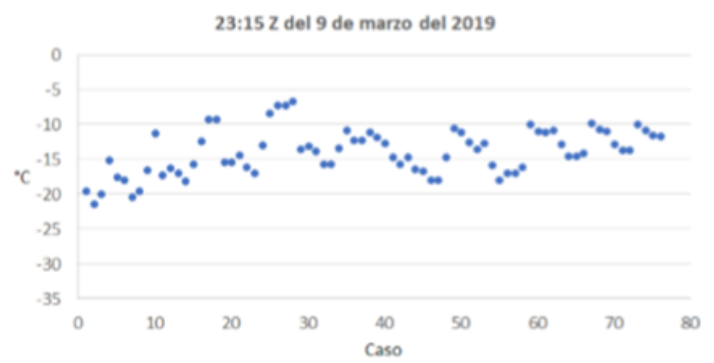

g. Estación: Santa Ana

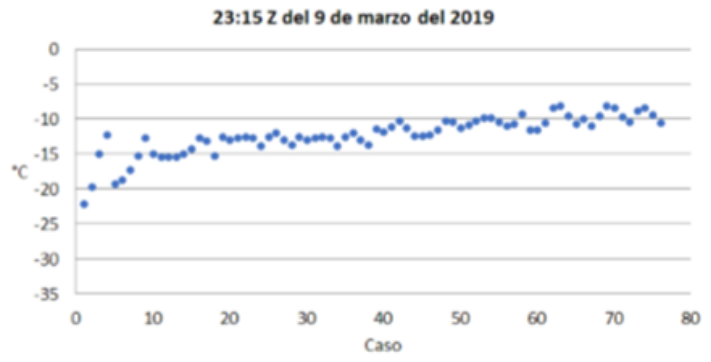

h. Estación: Tarma

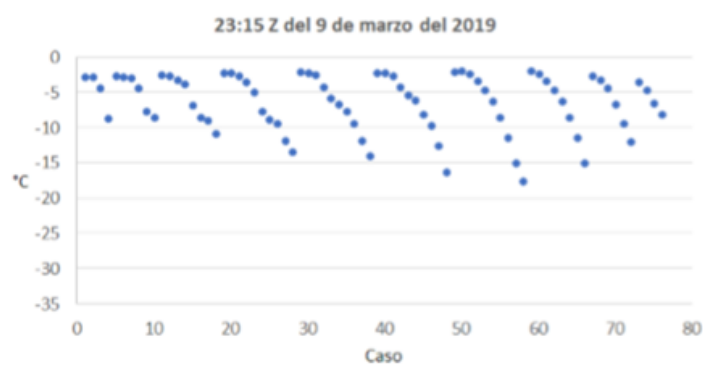

i. Estación: Yantac

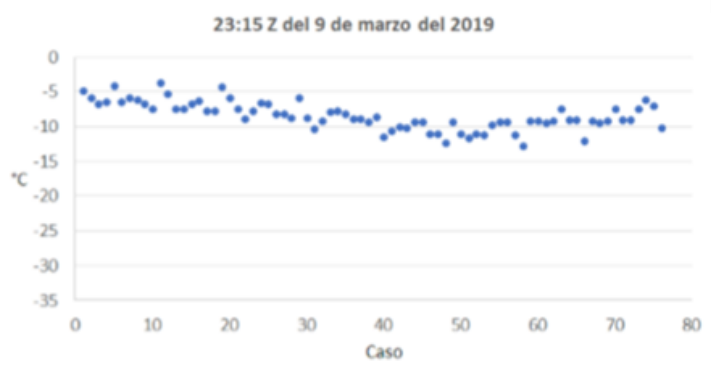

j. Estación: Jauja

Figura 7. Diferencia de temperatura de brillo (TB9-TB14) del satélite GOES 16 en estaciones de la cuenca del río Mantaro durante simulaciones. 09 de marzo del 2019. 


\footnotetext{
${ }^{1}$ Universidad Nacional Agraria La Molina. Av. La Universidad. s/n. La Molina. Lima - Perú. Lima 12.

2 Autor de correspondencia. wflores@lamolina.edu.pe.

3 Servicio Nacional de Meteorología e Hidrología. Jr. Cahuide 785. Jesús María. Lima - Perú. Lima 11. dacuna@senamhi.gob.pe.

${ }^{4}$ vcmquebec@lamolina.edu.pe.

5 jgarcia@lamolina.edu.pe.
} 